\title{
Weight reduction program with continuous psychological support in obese patients
}

\begin{abstract}
Background: Obese subjects have been increasing worldwide. Authors have continued clinical research about Low Carbohydrate Diet (LCD) and Calorie Restriction (CR) for long, and developed LCD medically and socially through the activity of Japan LCD Promoting Association (JLCDPA). Study protocol: Enrolled subjects enrolled were 2807 patients with obesity whom we gave advices in weight reduction program. Nutritional treatment on LCD was started by super-LCD with $12 \%$ of carbohydrate. After that, the carbohydrate limitation has been relieved according to the each situation of the subjects. Furthermore, subjects have received psychological supports with Empowerment, Motivation, and Medical Adherence (EMMA) and with the relationship among recognition of self-regulation; self-determination; conscientization and struggle were provided for 6-12 months.

Results and discussion: The weight changes of 2807 cases were studied with the following results: weight reduction rate of $10 \%<$ was observed in $691(32.3 \%), 5.0$ $9.9 \%$ was in $908(24.1 \%), 2.5-4.9 \%$ was in $593(21.1 \%)$. For totally calculated statistics data, reduction rate of $5.0 \%<$ was found in $56.4 \%$, and $2.5 \%<$ was $78.5 \%$. Current results seemed to be effective with LCD and psychological supports. These results would be one of the fundamental and referent data in this field of weight reduction for future research.
\end{abstract}

Keywords: weight reduction program, obesity low carbohydrate diet (LCD), empowerment, motivation, and medical adherence (EMMA), Japan LCD promoting association (JLCDPA)
Volume 9 Issue I - 2019

\author{
Bando $\mathrm{H},^{\prime}$ Nakamura T, ${ }^{2,3}$ Kawashima T, 2,3 \\ Dobashi $\mathrm{M},{ }^{2,3}$ Narita A, ${ }^{2,3}$ \\ 'Tokushima University / Medical Research,Tokushima, Japan \\ ${ }_{2}^{2}$ Japan Low Carbohydrate Diet Promotion Association \\ (JLCDPA), Kyoto, Japan \\ ${ }^{3}$ Nakamura Orthopedic Clinic and Anti-Aging Center, Kawanishi, \\ Hyogo, Japan
}

\begin{abstract}
Correspondence: Hiroshi Bando, MD, PhD, FACP,Tokushima University /Medical Research,Nakashowa I-6I, Tokushima 7700943 Japan, Tel +8I-90-3 I87-2485,

Email pianomed@bronze.ocn.ne.jp
\end{abstract}

Received: February 10, 2019 | Published: February 28, 2019
Abbreviation: LCD, low carbohydrate diet; CR, calorie restriction; RDN, registered dietitian nutritionists; JLCDPA, Japan LCD promoting association; EMMA, empowerment motivation medical adherence; T2DM, Type 2 diabetes mellitus

\section{Introduction}

Obesity has been recently one of the important public health problems, with the increasing tendency of Body Mass Index (BMI) in the world. ${ }^{1}$ The prevalence of obesity or overweight has been twice of that of 1980 and about one third of population in the world is supposed to be obese or overweight. ${ }^{2}$ The cause of the obesity has been multi-factorials, and the prevention of the obesity includes environmental interventions and various lifestyle changes. For the medical management of obesity and overweight, there was a standard guideline. It was the American College of Cardiology/ American Heart Association Task Force on Practice Guidelines and The Obesity Society (AHA/ACC/TOS) in 2013, and it showed various available evidence to medical practice. ${ }^{3}$ It has been often found that obese people had succeeded weight reduction, but could not maintain the weight easily. From previous reports, the important factors have been the correlations among lifestyle, dietary and psychosocial situations. ${ }^{4}$ Then, further research would be expected concerning the behavioral and clinical characteristics of maintaining satisfactory weight reduction. On the other hand, there was an 'adult disease' a few decades ago in Japan. After that, it was changed to 'life style related disease' because their geneses are probably due to inadequate lifestyle situation. ${ }^{5}$ It was proposed by Dr. Shigeaki Hinohara who was the President Emeritus of St. Luke International Hospital in Tokyo associated with the philosophy 'Hinohara-ism'. Consecutively, the medical term 'metabolic syndrome' has been prevalent including obesity, diabetes mellitus, hypertension and hyperlipidemia. There is a possibility that hyperuricemia and gout has been also added as another factors in the future. ${ }^{7}$ As to the treatment of metabolic syndrome, the basic therapy would be the control of the body weight. Consequently, the weight reduction should be tried at first for the treatment of metabolic syndrome and obesity prior to starting various medicine. ${ }^{8}$ The actual method for weight reduction includes both diet therapy and exercise therapy. For diet therapy, there was a standard method, which was calorie restriction (CR). After that, low carbohydrate diet (LCD) has been introduced in Western countries. In succession, authors and colleagues had started LCD in Japan ${ }^{9}$ and developed LCD through medical practice, medical society, workshop and textbooks. ${ }^{10}$ We have proposed three patterns of LCD meal including super-LCD, standard-LCD and petite-LCD, which can be applied in various clinical situation. ${ }^{11}$ Among our clinical research, we have treated lots of patients with obesity in weight reduction program. During the program, they were given medical, nutritional and psychological supports by medical staffs including registered dietitian nutritionists (RDNs). Their managements and advises were based on several medical and psychological supports. ${ }^{12}$ The detail of the program was described and discussed in this report.

\section{Patients and methods}

The subjects were the patients with obesity, who were treated in the weight reduction program of our clinic from 2003 to 2018. They have some other health problems in their background, including metabolic syndrome such as diabetes, hypertension, hyperlipidemia, and locomotive syndrome, flailty or joint problems. Methods in current study have several aspects, which are shown as follows.

\section{a) Intervention of nutritional therapy}

As to the diet therapy in our research, low carbohydrate diet (LCD) was applied to the subjects. We have our protocol of LCD therapy for the patients with obesity in the weight reduction program. There are 
three types of LCD application, which are super-LCD, standard-LCD, petite-LCD with carbohydrate ratio of $12 \%, 26 \%, 40 \%$, respectively. ${ }^{13}$

\section{b) Nutritional guidance of medical staffs}

For the nutritional therapy in the weight reduction program, medical staffs have advised the content in detail, including physicians, nurses, RDNs, pharmacists. Especially, RDNs became the main staffs to continue the diet management. In the first contact, we apply superLCD to the patients, so as to make the drastic change in the meal habit from previous pattern to the new one. The important period is two weeks from the beginning. With successful changed habit, the subjects would be advised to continue satisfactory process and results. After that, through frequent interviews between the patients and RDNs, adequate ratio of LCD would be consulted, advised, and continued. They include super-LCD, standard-LCD, petit-LCD, as well as $33 \%$ of carbohydrate pattern that is recommended specific ratio of anti-aging medical field by Grossman..$^{14,15}$

\section{c) Psychological supports of medical staffs}

In our weight reduction program, we have continued psychological supports for the patients who need some help in the process. They include the communication, consult, and advices which are based on the theory of Empowerment, Motivation, and Medical Adherence (EMMA $)^{16}$ Furthermore, an useful concept of educational and medical management was also applied to the advices, such as self-regulation, self-determination, and conscientization ${ }^{17}$ (Figure 1).

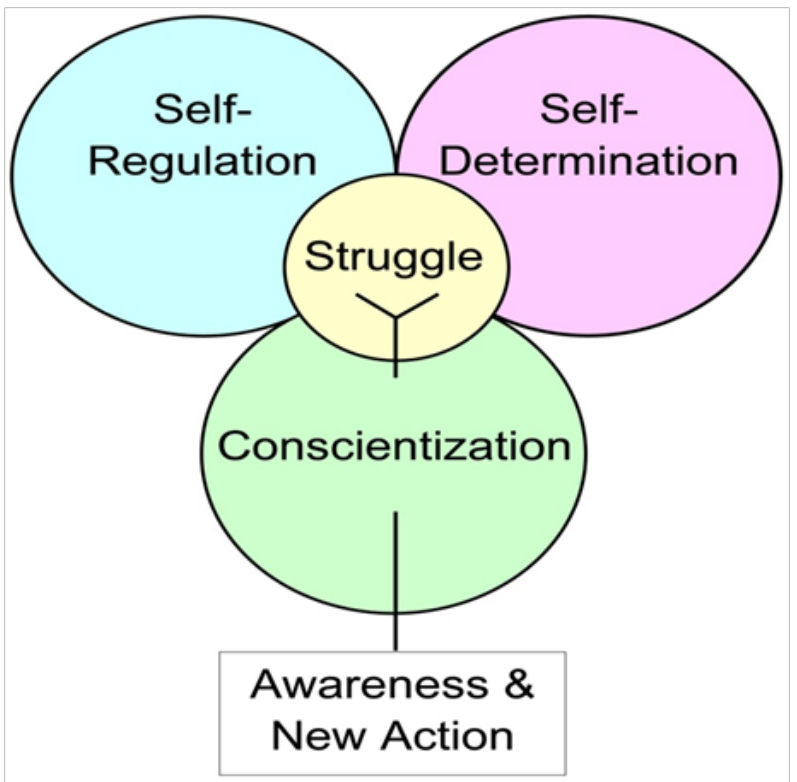

Figure I Recognition of the mind concerning self-regulation, selfdetermination and conscientization.

\section{d) Exercise therapy}

For exercise therapy, there are aerobic exercise and anaerobic exercise. We advised and managed the both exercises to the subjects. There is our fitness club adjacent to the clinic, where every person can use as they like. Subjects were always given adequate advice from physiotherapists, exercise trainers in the room. They are recommended to walk or jog using treadmill a few times a week. Moreover, subjects are advised to continue anaerobic training (resistance training) using the 11 kinds of equipment in the fitness room. The characteristic point of the training machine is not gravity-produced resistance, but fluid- resistant type exercising device. It is definite that this method is safer for the aged person and healthy non-athlete people. The series of the training machines are made of NewStep ${ }^{\mathrm{R}}$ and Transforming Lives ${ }^{\mathrm{R}}$ by Arthritis Foundation, Japan. ${ }^{18}$ Consequently, applying various apparatus, subjects were given aerobic and anaerobic exercise totally. ${ }^{19}$

\section{e) Weight changes}

Subjects enrolled were given diet therapy, psychological support and exercise mentioned above. The body weight was checked periodically, and before and after the intervention about 6-12 months later when the weight became stable. The comparison of the body weight was performed between pre-weight and post-weight. Both of them were used for calculation of the ratio of weight reduction.

\section{Results}

Weight reduction program has been continued. During the process, patients with obesity were followed up by medical staffs, including doctors, nurses, RDNs, pharmacists. The results from 2807 cases are revealed in Figure 2. There was decrease of body weight by the program as follows: weight reduction of $10 \%$ or more was observed in $691(32.3 \%)$, reduction of $5.0-9.9 \%$ was in $908(24.1 \%)$ and reduction of $2.5-4.9 \%$ was in $593(21.1 \%)$. When calculated together, reduction rate of $5.0 \%$ or more was observed in $56.4 \%$, and reduction of $2.5 \%$ or more was $78.5 \%$.

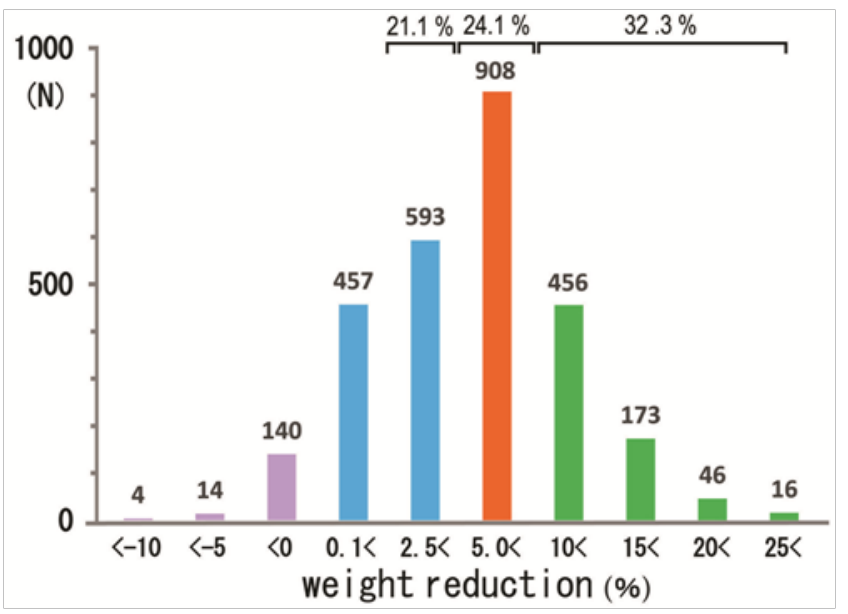

Figure 2 Reduction rate of the body weight in 2807 subjects.

\section{Discussion}

According to the previous reports, there are some points or characteristics which successfully brought weight reduction. ${ }^{20}$ They showed the same tendencies reported by successfully weight-reduced subjects who attended the professional-led educational program. Some factors were more effective such as

i) Earlier embedding for the new lifestyle changes into daily routine habit,

ii) Learning from their previous weight reduction methods and experiences,

iii) Not necessary for the existence of various social supports. ${ }^{20}$ especially, the probable factors leading to successful results would be problem solving skills and thinking, self-efficacy and self-reliance, and continuing internal motivation. 
As to the issue of adherence for the person seeking to weight reduction, persistence of long term dietary changes are not so easy. ${ }^{21}$ According to the method of the Interpretative Phenomenological Analysis (IPA), nine factors were identified. They are

1) Professional management,

2) Thorough and deep achievement,

3) Accepting the result as it is,

4) Persisting care by medical staff,

5) Recognition of the person in main part of the process,

6) Empowerment by necessary knowledge,

7) Adequate shift in focus to better health,

8) Continuing the motivation, and

9) Emphasizing what the person have to do. These factors would be classified into four groups, which are providing coaching and education, enhancing professionalism and their relationship with the subjects in charge. ${ }^{21}$ As to the relationship about evidence and adherence, there are 4 phases. They are

a. Evidence of change,

b. Experience of mastery,

c. Increased self-efficacy and

d. Encouraging adherence to program. These factors will be continuing in the cyclic manner. ${ }^{21}$

When the subjects have the successful results with weight reduction and symptom improvement, these can be classified as Experience of Mastery and Enactive Attainment. They are characteristic for the theory of Bandura concerning self-efficacy. ${ }^{22}$ further, the relationship among self-efficacy; adherence and weight loss are important problems. If the subjects recognize that the weight reduction program can bring the preferable results, they can encourage themselves to believe the obtained mastered skill for weight reduction. ${ }^{23}$ These processes may increase the degree of self-efficacy and also encourage the development of adherence. Subjects who have obtained successful weight reduction tended to show psychologically controlled status. On the process of continuing diet therapy, every person feels some struggles. In such case, there are three concepts in the mind, which are self-regulation, self-determination, and conscientization. ${ }^{17}$ (Figure 2). From these aspects, medical staffs must listen carefully, encourage and advise the patients. In our clinical practice, medical staffs always communicate and consult together for the pursuit of successful and satisfactory weight reduction. There was a report of Empowerment, Motivation, and Medical Adherence (EMMA) research for Type 2 diabetes mellitus (T2DM). ${ }^{16}$ EMMA includes a patient-oriented approach by dialogue tools in order to help self-reflection, learning and goal-setting for 3-4 months. Furthermore, they investigated the questionnaire scores for self-determination theory, using autonomy support Health Care Climate Questionnaire (HCCQ). Thus, various aids for successful weight reduction can be applied through the interrelationship between the patients and medical staffs. We can use a reference book to be helpful for better weight reduction. ${ }^{24}$ There are some limitations in this report. Indeed, we have treated many obese patients in the program, but the managements and advises were different according to the situation of each subject. Actually, two groups cannot be compared whether the empowerment management was performed or not. ${ }^{25}$ These problems would be considered and investigated in the future. In summary, the results of weight reduction program in lots of obese patients were described. Furthermore, psychological supports such as EMMA associated by medical staffs seemed to be effective for weight reduction and maintenance. These data would become the fundamental reference for the future study.

\section{Acknowledgments}

None.

\section{Conflicts of interest}

The authors declared there is no conflict of interest.

\section{References}

1. Caballero B. Humans against Obesity: Who Will Win?, Adv Nutri. 2019;10(1)suppl1:S4-S9.

2. ChooiYC, Ding C, Magkos F. The epidemiology of obesity. Metabolism. 2018;92:6-10.

3. Jensen MD, Ryan DH, Apovian CM, et al. 2013 AHA/ACC/TOS guideline for the management of overweight and obesity in adults: a report of the American College of Cardiology/American Heart Association Task Force on Practice Guidelines and The Obesity Society. Circulation. 2014;129(25):S102-138.

4. Poulimeneas D, Yannakoulia M, Anastasiou C, et al. Weight Loss Maintenance: Have We Missed the Brain? Brain Sci. 2018;8(9):174.

5. Fukui T, Rahman M, Ohde S, et al. Reassessing the ecology of medical care in Japan. J Community Health. 2017;42:935-941.

6. Hinohara S. A proposal for new term habit disease instead of adult disease. Educ Health Serv. 1978;5: 1-2.

7. Genoni G, Menegon V, Secco GG, et al. Insulin resistance, serum uric acid and metabolic syndrome are linked to cardiovascular dysfunction in pediatric obesity. Int J Cardiol. 2017;249:366-371.

8. Pearl RL, Wadden TA, Hopkins CM, et al. Association between weight bias internalization and metabolic syndrome among treatment-seeking individuals with obesity. Obesity (Silver Spring). 2017;25(2):317-322.

9. Ebe K, Ebe Y, Yokota S, et al. Low Carbohydrate diet (LCD) treated for three cases as diabetic diet therapy. Kyoto Medical Association Journal. 2004;51:125-129.

10. Nakamura T, Bando H. Low carbohydrate diet (LCD) has to be applied for nutritional therapy. The Journal of the Therapy. 2009;91:2858-2859.

11. Bando H, Ebe K, Muneta T, et al. Difference of Glucose variability between Low Carbohydrate Diet (LCD) and Calorie Restriction (CR). Asp Biomed Clin Case Rep. 2018;1(2):4-15.

12. Armstrong MJ, Mottershead TA, Ronksley PE, et al. Motivational interviewing to improve weight loss in overweight and/or obese patients: a systematic review and metaanalysis of randomized controlled trials. Obes Rev. 2011;12(9):709-723.

13. Bando H, Ebe K, Muneta T, Bando M, Yonei Y. Clinical Effect of Low Carbohydrate Diet (LCD): Case Report. Diabetes Case Rep. 2017;2:124

14. Grossman T. Latest advances in antiaging medicine. Keio $J$ Med. 2005;54(2):85-94.

15. Feinman RD, Pogozelski WK, Astrup A, et al. Dietary carbohydrate restriction as the first approach in diabetes management: Critical review and evidence base. Nutrition. 2015;31(1):1-13.

16. VarmingAR, Olesen K, Willaing I. Empowerment, Motivation, and Medical Adherence (EMMA) - Results of an RCT in Patients with Poorly Regulated Type 2 Diabetes. Diabetes. 2018;67(Suppl 1). 
17. Diaz-GreenbergR, Thousand J, Cardelle-Elawar M, et al. What teachers need to know about the struggle for self-determination (conscientization) and self-regulation: adults with disabilities speak about their education experiences. Teaching and Teacher Education. 2000;16(8):873-887.

18. Pilutti LA, Paulseth JE, Dove C, et al. Exercise Training in Progressive Multiple Sclerosis. A Comparison of Recumbent Stepping and Body Weight-Supported Treadmill Training. Int J MS Care. 2016;18:221229.

19. Rafiei N, Gill T. Identification of factors contributing to successful self-directed weight loss: a qualitative study. J Hum Nutr Diet. 2017; 31(3):329-336

20. Miles HGE, Barrow M. Committed to Weight Loss: an IPAAnalysis Into the Experiences of Individuals Who Lost Weight Through Nutritional Intervention. Curr Res Nutr Food Sci. 2018;6(1).
21. Bandura A. Self-Efficacy: The Exercise of Control. New York: Freeman \& Co Ltd. 1997.

22. Shay LE. A Concept Analysis: Adherence and Weight Loss. Nursing Forum. 2008;43(1):42-52.

23. Graham K. Weight loss empowerment "50 way to lose your luggage". Grande Gringos, Inc. 2010.

24. Lee SJ. An Empowerment Program to Improve Self-Management in Patients with Chronic Kidney Disease. Korean $J$ Adult Nurs. 2018;30(4):426-436. 